\title{
The Formation of Addiction Psychotherapy Core Can be a Suit Supplement to the Methadone/Buprenorphine Maintenance Therapy Centers: A Fresh Bid to Successfully Treat Drug Abuse
}

\author{
Saeid Komasi, ${ }^{1}$ Mozhgan Saeidi, ${ }^{2}$ and Ali Soroush ${ }^{3,}{ }^{*}$ \\ ${ }^{1}$ Clinical Research Development Center, Imam Reza Hospital, Kermanshah University of Medical Sciences, Kermanshah, IR Iran \\ ${ }^{2}$ Cardiac Rehabilitation Center, Imam Ali Hospital, Kermanshah University of Medical Sciences, Kermanshah, IR Iran \\ ${ }^{3}$ Lifestyle Modification Research Center, Imam Reza Hospital, Kermanshah University of Medical Sciences, Kermanshah, IR Iran \\ "Corresponding author: Ali Soroush, Lifestyle Modification Research Center, Imam Reza Hospital, Kermanshah University of Medical Sciences, Zakaria Razi Boulevard, \\ Kermanshah, IR Iran. Tel: +98-8334276299, Fax: +98-8334276299, E-mail: alisoroush.kums@yahoo.com
}

Received 2017 February 28; Revised 2017 April 15; Accepted 2017 April 16.

Keywords: Methadone/Buprenorphine Maintenance Therapy, Drug Abuse, Psychotherapy

\section{Dear Editor,}

During recent years, methadone/buprenorphine maintenance therapy (MMT/BMT) has been one of the effective methods in controlling substance abuse and risky behaviors in Iran $(1,2)$. However, one of the major challenges of this therapeutic approach is treatment retention rate (3). Obviously, there are weaknesses in the routine work of these centers that increase the likelihood of treatment failure (4). For example, at least 50 patients are undergoing methadone therapy in Grade 4 clinics and some patients are under treatment with buprenorphine and opium tincture. However, in each of MMT/BMT clinics, only one psychologist is active who usually does not have enough experience. Additionally, according to the working hours of the clinics, the psychologist does not have enough time to provide exclusive psychotherapy. On the other hand, today in developed countries, the emphasis is placed on specialization in implement interventions. Specialization or specialized interventions mean that each part of the treatment is processed to be performed by a qualified and experienced professional. As a result, the multi-component psychiatric profile of patients treated in centers has required implementing the specialized interventions by an experienced psychotherapy team (5). Thus, it seems that specialized interventions must be performed by a number of clinical psychologists and psychiatrists because the patients can benefit from the specialized and extensive services of the psychotherapy team.

Given these considerations, the formation of a number of addiction psychotherapy cores in every city with the participation of various experts can be fruitful. So that in each metropolitan area that covers a number of MMT/BMT clinics, an addiction psychotherapy core can be formed. Then, expert psychologists in the field of individ- ual psychotherapy, couples and family therapy, and treatment of personality and clinical disorders can be placed in each of these cores (5). Also, a psychiatrist can join this group for the diagnosis of psychosis and drug therapy which would be beneficial (5). It seems that through this method, MMT/BMT centers' psychologists can focused solely on the initial interview, motivational interview, and supportive psychotherapy which may create better efficiency (4). Moreover, referral of the patients to the addiction psychotherapy core, giving diagnostic interviews, and receiving appropriate and timely treatment can prevent the wandering of the patients and their family members (6). This procedure reduces the likelihood of dropping out of treatment and re drug abuse (3). Therefore, we recommend the formation of addiction psychotherapy cores that simultaneously cover a number of MMT/BMT clinics. Our suggestive model is shown in Figure 1.

\section{Footnotes}

Authors' Contribution: All authors participated in the design of the study and drafted the manuscript and read and approved the final manuscript.

Conflict of Interest: None of the authors have conflicts of interest to report.

Funding/Support: None.

Competing Interests: None declared. 


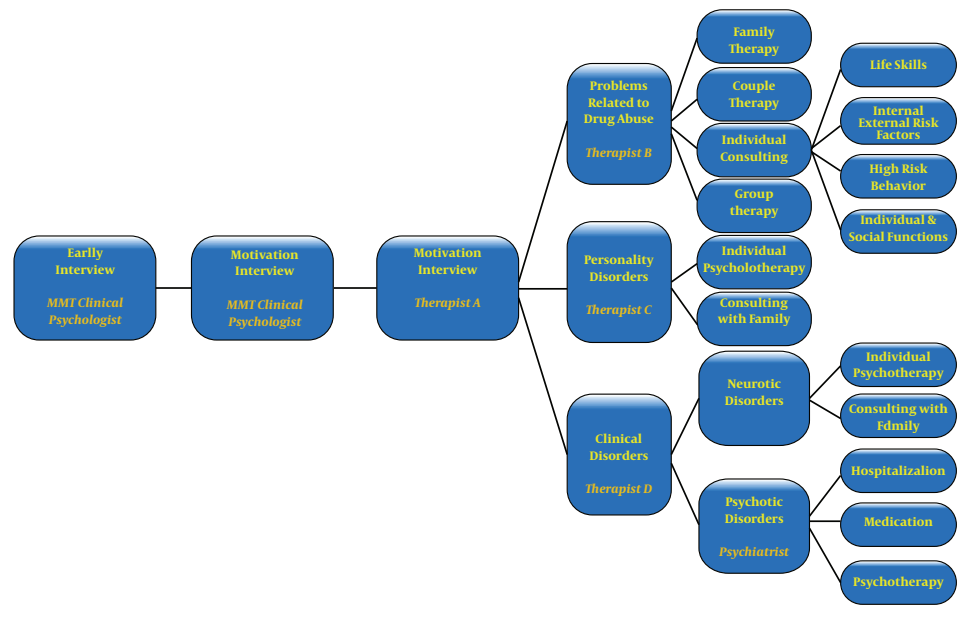

Figure 1. The Suggestive Model. MMT:methadone maintenance therapy.

\section{References}

1. Montazerifar F, Karajibani M, Lashkaripour K. Effect of methadone maintenance therapy on anthropometric indices in opioid dependent patients. Int J High Risk Behav Addict. 2012;1(3):100-3. doi: 10.5812/ijhrba.4968. [PubMed: 24971244].

2. Kheradmand A, Banazadeh N, Abedi H. Physical effects of methadone maintenance treatment from the standpoint of clients. Addict Health. 2010;2(3-4):66-73. [PubMed: 24494103].

3. Meier PS, Donmall MC, McElduff P, Barrowclough C, Heller RF. The role of the early therapeutic alliance in predicting drug treatment dropout. Drug Alcohol Depend. 2006;83(1):57-64. doi: 10.1016/j.drugalcdep.2005.10.010. [PubMed:16298088].
4. Alam-Mehrjerdi Z, Abdollahi M. The Persian methamphetamine use in methadone treatment in Iran: implication for prevention and treatment in an upper-middle income country. Daru. 2015;23:51. doi: 10.1186/s40199-015-0134-5. [PubMed: 26578071].

5. Dugosh K, Abraham A, Seymour B, McLoyd K, Chalk M, Festinger D. A Systematic Review on the Use of Psychosocial Interventions in Conjunction With Medications for the Treatment of Opioid Addiction. J Addict Med. 2016;10(2):93-103. doi: 10.1097/ADM.0000000000000193. [PubMed: 26808307].

6. Friedmann PD, Lemon SC, Stein MD, D’Aunno TA. Accessibility of addiction treatment: results from a national survey of outpatient substance abuse treatment organizations. Health Serv Res. 2003;38(3):887903. [PubMed: 12822917]. 